\title{
On Fuzzy Soft Set-Valued Maps with Application
}

\author{
Mohammed Shehu Shagari* \\ Department of Mathematics, Faculty of Physical Sciences, Ahmadu Bello University, Nigeria
}

\begin{abstract}
Soft set and fuzzy soft set theories are proposed as mathematical tools for dealing with uncertainties. There has been tremendous progress concerning the extensions of these theories from different point of views of researchers so as to accommodate more robust and expressive applications in everyday life. In line with this development, in this paper, we combine the two aforementioned notions to initiate a novel concept of set-valued maps whose range set is a family of fuzzy soft sets. The later idea is employed to define Suzuki-type fuzzy soft $(e, K)$-weak contractions, thereby establishing some related fuzzy soft fixed point theorems. As a consequence, several well-known Suzuki-type fixed point theorems are derived as corollaries. Examples are also provided to validate the new concepts and to support the authenticity of the obtained results. Moreover, an application in homotopy is considered to show the usability of the obtained results.
\end{abstract}

Keywords: Soft set, Fuzzy soft set, Fuzzy soft set-valued map, Fuzzy soft fixed point, Weak contraction

Article History :

Received: 30 January 2020

Received in revised form: 14 February 2020

Accepted for publication: 15 February 2020

Published: 28 February 2020

(C)2020 Journal of the Nigerian Society of Physical Sciences. All rights reserved.

Communicated by: B. J. Falaye

\section{Introduction and preliminaries}

The classical Banach contraction theorem [1] is one of the extremely useful results in nonlinear functional analysis. In the setting of a metric space, this theorem is stated as follows.

Theorem 1.1. [1] Let $(X, d)$ be a complete metric space and $T: X \longrightarrow X$ be a contraction, i.e., there exists an $\alpha \in(0,1)$ such that

$$
d(T x, T y) \leq \alpha d(x, y), \text { for all } x, y \in X .
$$

\section{Then}

(i) Thas a unique fixed point $u$ in $X$;

\footnotetext{
${ }^{*}$ Corresponding author tel. no:

Email address: ssmohammed@abu.edu.ng (Mohammed Shehu Shagari)
}

(ii) The Picard iteration $\left\{x_{n}\right\}_{n=0}^{\infty}$, given by

$$
x_{n+1}=T x_{n}, n=0,1,2, \cdots
$$

converges to $u$, for any $x_{0}$ in $X$.

Theorem 1.1 has several generalizations, see, for example, $[2$, $3,4,5,6,7]$ and the references therein. The two well-known drawbacks of Theorem 1.1 are:

(drb1) The contractive condition in (1) compels $T$ to be continuous;

(drb2) The theorem cannot characterize metric completeness of $X$.

Problems (drb1)-(drb2) were resolved affirmatively by Kannan [18]. Recall that a mapping $T$ (not necessarily continuous) on a metric space $X$ is said to be a Kannan contraction if there exists an $\alpha \in\left[0, \frac{1}{2}\right)$ such that

$$
d(T x, T y) \leq \alpha[d(x, T x)+d(y, T y)], \text { for all } x, y \in X .
$$


This achievement in Kannan contraction was first noted by Subrahmanyam [28] that a metric space $X$ is complete if and only if every Kannan contraction on $X$ has a fixed point. Following (2), more than a handful of papers were devoted to studying fixed point theorems for classes of contractive type conditions that do not require the continuity of $T$; see, for instance, [8, 9, $10,11,12]$. However, researchers noticed that Kannan's theorem is not a generalization of Theorem 1.1. Along the way, the notion of weak contraction was introduced by Berinde [13]. The idea generalized the well-celebrated fixed point theorems due to Banach [1], Chatterjea [9], Zamfirescu [12], and many others.

Definition 1.2. Let $(X, d)$ be a metric space. A mapping $T$ : $X \rightarrow X$ is called weak contraction if there exists a constants $\alpha \in(0,1)$ and $K \geq 0$ such that

$$
d(T x, T y) \leq \alpha d(x, y)+K d(y, T x), \text { for all } x, y \in X .
$$

Thereafter, in 2007, M.Berinde and V. Berinde [14] extended the concept of weak contraction from the case of single-valued mappings to multi-valued mappings and established some convergence theorems for the Picard iteration in connection with multi-valued weak contraction.

Definition 1.3. Let $(X, d)$ be a metric space and $T: X \longrightarrow$ $C B(X)$ be a multi-valued mapping. Then $T$ is called a multivalued weak contraction or multi-valued $(\theta, L)$-weak contraction if and only if there exist constants $\theta \in(0,1)$ and $K \geq 0$ such that for all $x, y \in X$,

$$
H(T x, T y) \leq \theta d(x, y)+L d(y, T x) .
$$

Furthermore, a notable attempt at resolving problems (drb1)(drb2) was presented in 2008 by Suzuki [29]. The following is known in the literature as Suzuki fixed point theorem.

Theorem 1.4. Let $(X, d)$ be a complete metric space and $T$ be a mapping on $X$. Define a nondecreasing function $r$ from $[0,1)$ onto $\left(\frac{1}{2}, 1\right]$ by

$$
r(t)= \begin{cases}1, & \text { if } 0 \leq t \leq \frac{\sqrt{5}-1}{2} \\ \frac{(1-t)}{r^{2}}, & \text { if } \frac{\sqrt{5}-1}{2} \leq r \leq \frac{1}{\sqrt{2}} \\ \frac{1}{(1+t)}, & \text { if } \frac{1}{\sqrt{2}} \leq r<1 .\end{cases}
$$

Assume that there exists $t \in[0,1)$ such that

$$
r(t) d(x, T x) \leq d(x, y)
$$

implies

$$
d(T x, T y) \leq r d(x, y), \text { for all } x, y \in X .
$$

Then $T$ has a unique fixed point $u$ in $X$. Moreover, $T^{n} x \longrightarrow u$ as $n \longrightarrow \infty$ for all $x \in X$.

An interesting improvement of Suzuki fixed point theorem in the setting of multivalued mappings is due to Djoric and Lazovic [16]. In subsequent section, we shall derive the main result in [16] as a consequence of our result.
The real world is filled with uncertainty, vagueness and imprecision. The notions we meet in everyday life are vague rather than precise. In recent time, researchers have taken keen interests in modelling vagueness due to the fact that many practical problems within fields such as biology, economics, engineering, environmental sciences, medical sciences involve data containing various forms of uncertainties. To handle these complications, one cannot successfully employ classical mathematical methods due to the presence of different kinds of incomplete knowledge typical of these mix-ups. Earlier in the literature, there are four known theories for dealing with imperfect knowledge, namely Probability Theory (PT), Fuzzy Set Theory (FST)[30], Interval Mathematics and Rough Set Theory (RST)[27]. All the aforementioned tools require pre-assignment of some parameters; for example, membership function in FST, probability density function in PT and equivalent relation in RST. Such pre-specifications, viewed in the backdrop of incomplete knowledge, give rise to every day problems. In this concern, Molodstov [26] initiated the concept of Soft Set Theory (SST) with the aim of handling phenomena and notions of ambiguous, undefined and imprecise environments. Hence, SST does not need the pre-specifications of a parameter, rather it accommodates approximate descriptions of objects. In other words, one can use any suitable parametrization tool with the help of words, sentences, real numbers, mappings, and so on. Thereby, making SST an adequate formalism for approximate reasoning. In the pioneer work of Molodstov [26], several potential applications of SST have been pointed out in the areas of Riemann integration, smoothness of functions, theory of probability, theory of measurement, game theory and operation research . Interestingly, Molodstov [26] emphasized that the models by fuzzy sets and soft sets are interrelated. Yang et al [22] emphasized that SST needed to be expanded in different directions to extend its applications to other fields. Along the lane, by combining the ideas of soft sets and fuzzy sets, Maji et al [21] initiated the concept of fuzzy soft sets and discussed its various properties. Recent researches [20, 21, 22] have shown that both theories of FST and SST can be combined to have a more flexible and expressive framework for modelling and processing data and information possessing nonstatistical uncertainties.

In this paper, by combining the ideas of soft sets and fuzzy soft sets, the concept of fuzzy soft set-valued maps is initiated; that is, a map whose range set is a family of fuzzy soft sets. Thereafter, motivated by the work of Suzuki [29], Djoric and Lazovic [16], we define the notion of Suzuki-type fuzzy soft $(e, K)$-weak contraction in the setting of fuzzy soft sets, where $e$ is an element of the parameter set $E$ and $K \geq 0$. As a result, fuzzy soft fixed point theorem of Suzuki-type is proved and some consequences are obtained thereafter. In addition, an application in homotopy result is established to show the usability of our obtained results.

The rest of this paper is organized as follows. In Subsection 1.1 , specific concepts of fuzzy sets and multivalued mappings are recalled. Subsection 1.2 briefly reviews some background of soft sets and fuzzy soft sets. Section 2 contains the novel concepts of fuzzy soft set-valued maps. In Subsection 2.1, a 
few fuzzy soft fixed point theorems of Suzuki-type $(e, K)$-weak contractions are established. Subsection 2.2 gives some consequences of Subsection 2.1. An application in homotopy result is presented in Subsection 2.3.

\subsection{Fuzzy Sets and Multivalued Mappings}

Let $(X, d)$ be a metric space. We denote by $C B(X)$ the class of all nonempty, closed and bounded subsets of $X$. Let $H(.,$. be the Hausdorff metric on $C B(X)$ induced by $d$, that is,

$$
H(A, B)=\max \left\{\sup _{a \in A} d(a, B), \sup _{b \in B} d(A, b)\right\},
$$

for $A, B \in C B(X)$, where $d(x, A)=\inf \{d(x, a): a \in A\}$. A point $u$ in $X$ is a fixed point of a multi-valued mapping $T: X \longrightarrow$ $C B(X)$ if $u \in T u$.

Let $X$ be an initial universe. Recall that an ordinary subset $A$ of $X$ is determined by its characteristic function $\chi_{A}$, defined by $\chi_{A}: A \longrightarrow\{0,1\}$ :

$$
\chi_{A}(x)= \begin{cases}1, & \text { if } x \in A \\ 0, & \text { if } x \notin A .\end{cases}
$$

The value $\chi_{A}(x)$ specifies whether an element belongs to $A$ or not. This idea is used to define fuzzy sets by allowing an element $x \in A$ to assume any possible value in the interval $[0,1]$. Thus, a fuzzy set $A$ in $X$ is a set of ordered pair given as

$$
A=\left\{\left(x, \mu_{A}(x)\right): x \in X\right\},
$$

where $\mu_{A}: X \longrightarrow[0,1]=I$ and $\mu_{A}(x)$ is called the membership function of $x$ or the degree to which $x \in X$ belongs to the fuzzy set $A$. If $A$ is a fuzzy set in $X$, the (crisp) set of elements in $X$ belonging to $A$ at least of degree $\alpha \in(0,1]$ is called the $\alpha$-level set, denoted by $[A]_{\alpha}$. That is,

$$
[A]_{\alpha}=\left\{x \in X: \mu_{A}(x) \geq \alpha\right\} .
$$

On the other hand,

$$
[A]_{\alpha}^{*}=\left\{x \in X: \mu_{A}(x)>\alpha\right\},
$$

is called the strong $\alpha$-level set or strong $\alpha$-level cut. Denote by $I^{X}$, the family of all fuzzy sets in $X$. A mapping $T: X \longrightarrow I^{X}$ is called fuzzy mapping. An element $u$ in $X$ is said to be a fuzzy fixed point of a fuzzy mapping $T$ if there exists an $\alpha \in$ $(0,1]$ such that $u \in[T u]_{\alpha}$. If there exists $\alpha \in[0,1]$ such that $[A]_{\alpha},[B]_{\alpha} \in C B(X)$, then

$$
d_{\infty}(A, B)=\sup _{\alpha} H\left([A]_{\alpha},[B]_{\alpha}\right) .
$$

\subsection{Soft Sets and Fuzzy Soft Sets}

Let $E$ be the parameter set, $A \subseteq E$ and $P(X)$ represents the power set of an initial universe of discourse $X$. In this section, some basic concepts and examples of soft sets and fuzzy soft sets are recalled.

Molodstov [26] established the concept of soft sets with the following definition.
Definition 1.5. [26] A pair $(F, A)$ is called a soft set over $X$ under $E$, where $A \subseteq E$ and $F$ is a mapping given by $F: A \longrightarrow$ $P(X)$.

In other words, a soft set over $X$ is a parameterized family of subsets of $X$. For each $e \in E, F(e)$ is considered as the set of $e$-approximate elements of $(F, A)$.

Example 1.6. [25] Suppose the following:

$X$ - is the universal set of all students in a certain university, $E$ - is the set of parameters, given as:

$$
E=\{\text { intelligent, hardworking, dull, hardworking and intelligent }\} .
$$

Assume that they are one hundred students in the university $X$ given as

$$
X=\left\{x_{1}, x_{2}, x_{3}, x_{4}, x_{5} \cdots x_{100}\right\}, \quad \text { and } E=\left\{e_{1}, e_{2}, e_{3}, e_{4}\right\},
$$

where

$$
\begin{aligned}
& e_{1}=\text { intelligent }, \quad e_{2}=\text { hardworking, } \\
& e_{3}=\text { dull, } \quad e_{4}=\text { hardworking and intelligent } .
\end{aligned}
$$

Then $F: E \longrightarrow P(X)$ defined by $F\left(e_{1}\right)=\left\{x_{1}, x_{2}, \cdots x_{10}\right\}$ means that $x_{1}, x_{2}, \cdots x_{10}$ are intelligent, $F\left(e_{2}\right)=\left\{x_{11}, x_{12}, \cdots x_{30}\right\}$ means that $x_{11}, x_{12}, \cdots x_{30}$ are hardworking, $F\left(e_{3}\right)=\emptyset$ means that there is no dull student in the university in question, $F\left(e_{4}\right)=$ $\left\{x_{15}, x_{81}\right\}$ means that the students $x_{15}$ and $x_{81}$ are both intelligent and hardworking. Then we can view the soft set $(F, E)$ describing the "kind of students" as the following approximations:

$$
\begin{aligned}
(F, E)= & \left\{\text { (intelligent students, }\left\{x_{1}, x_{2}, \cdots x_{10}\right\}\right), \\
& \text { (hardworking studnts, } \left.\left\{x_{11}, x_{12}, \cdots x_{30}\right\}\right)
\end{aligned}
$$

(dull, $\emptyset$ ), (intelligent and hardworking students, $\left.\left.\left\{x_{15}, x_{81}\right\}\right)\right\}$.

Many researchers carried out formal studies of these basic ideas of soft sets and related notions. For example, Maji etal [24] developed these notions and established other concepts such as soft subsets and supersets, intersections and unions, soft equalities and so on. For soft set-based decision making approach, the interested reader may consult Cagman and Enginoglu [15], Feng and Zhou[17] and Maji et al [23].

Remark 1.7. (See [26]) Every fuzzy set is a special kind of soft set. In other words, let A be a fuzzy set with membership function $\mu_{A}$. Consider the family of $\alpha$-level sets given by

$$
\Omega(\alpha)=\left\{x \in X: \mu_{A}(x) \geq \alpha\right\}, \quad \alpha \in[0,1] .
$$

If the family $\Omega$ is known, then we can find $\mu_{A}(x)$ via the formula:

$$
\mu_{A}(x)=\sup \{\alpha: \alpha \in[0,1]\}, \quad x \in \Omega(\alpha) .
$$

This shows that in particular, every fuzzy set is a soft $\operatorname{set}(\Omega,[0,1])$.

In order to model more general scenarios, Maji et al [21] defined the notion of fuzzy soft sets in the following manner. 
Definition 1.8. [21] A pair $(F, A)$ is a fuzzy soft set over $X$ when $A \subseteq E$ and $F: A \longrightarrow I^{X}$.

Clearly, every soft set can be thought of as a fuzzy soft set. Following Example 1.6, fuzzy soft sets allow the investigation of some more intriguing properties such as "how much time each student works" in which case partial memberships are indispensable.

Example 1.9. [25] Consider Example 1.6. The fuzzy soft set $(F, E)$ describing the "kind of students" under fuzzy circumstances may be given as

(i) $F\left(e_{1}\right)=\left\{x_{1} / 0.5, x_{2} / 0.1, x_{4} / 0.7\right\}$, $F\left(e_{2}\right)=\left\{x_{3} / 0.6, x_{9} / 0.7, x_{20} / 0.1\right\}$

(ii) $F\left(e_{3}\right)=\left\{x_{19} / 0.8, x_{25} / 0.1, x_{4} / 0.7\right\}$, $F\left(e_{4}\right)=\left\{x_{13} / 0.4, x_{91} / 0.3, x_{94} / 0.1, x_{97} / 0.3\right\}$.

In what follows, we initiate the concept of fuzzy soft setvalued maps.

\section{Main Results}

Let $X$ be an initial universe of discourse, $E$ the parameter set, $I=[0,1]$ and $I^{(E ; X)}$ denotes the family of fuzzy soft sets over $X$ under $E$. By $A \in I^{(E ; X)}$, we mean the mapping $A: E \longrightarrow$ $I^{X}$, where $X$ is the set of all fuzzy sets in $X$. Let $\mathbb{N}, \mathbb{Z}$, and $\mathbb{R}$ denote the set of nonnegative integers, set of integers and the set of real numbers, respectively.

Definition 2.1. A mapping $T: X \longrightarrow I^{(E ; X)}$ is called fuzzy soft set-valued map.

Notice that if $T: X \longrightarrow I^{(E ; X)}$ is a fuzzy soft set-valued map, then for $x \in X, T x \in I^{(E ; X)}$ implies that $T x: E \longrightarrow I^{X}$ is a fuzzy soft set. This further implies that for any $e \in E$, $T(x)(e): X \longrightarrow[0,1]$ is a fuzzy set. Hence, $(T x)(e)(t)$ is the degree of membership of $t$ in $(T x)(e)$. In the remaining part of this paper, we shall write $T[x ; e]$ and $T[x ; e](t)$ to represent $(T x)(e)$ and $(T x)(e)(t)$, respectively.

Example 2.2. Let $X=[-4,4]$ and $E=[0,5]$. Define $T: X \longrightarrow$ $I^{(E ; X)}$ by

$$
T[x ; e](t)=\frac{t^{2}}{x^{2}+e+t^{2}} .
$$

Then $T$ is a fuzzy soft set-valued map. Notice that $T[x ; e](t) \in$ $[0,1]$ for all $x, t \in X$ and $e \in E$.

Definition 2.3. Let $T: X \longrightarrow I^{(E ; X)}$ be a soft set-valued map. Then the e-level set of $T$ is defined by

$$
[T x]_{E}^{e}=\bigcup_{e \in E}[T[x ; e]]_{E}^{e},
$$

where

$$
[T[x ; e]]_{E}^{e}=\{t \in X: T[x ; e](t) \geq e\}, e \in E .
$$

For simplicity, we shall also denote the e-level set of $T$ by $[T]_{E}^{e}$, whenever the domain of $T$ is obvious.
Proposition 2.4. Every $\alpha$-level set is an e-level set.

Proof. Let $A$ be a fuzzy set in $X$ and $E=(0,1]$ be a set of parameters. Consider a fuzzy soft set-valued map $T: X \longrightarrow$ $I^{(E ; X)}$ defined by

$$
T[x ; e](t)=A(x) \text {, for all } x, t \in X \text { and } e \in E .
$$

Then for $\alpha \in(0,1]=E$, we have

$$
\begin{aligned}
\bigcup_{\alpha \in(0,1]}[A]_{\alpha} & =\bigcup_{\alpha \in(0,1]}\{t \in X: A(x) \geq \alpha\} \\
& =\bigcup_{\alpha \in(0,1]}\{t \in X: T[x ; e](t) \geq \alpha=e\} \\
& =\bigcup_{e \in E}\{t \in X: T[x ; e](t) \geq e\} \\
& =[T]_{E}^{e} .
\end{aligned}
$$

Remark 2.5. Every fuzzy mapping $\Upsilon: X \longrightarrow I^{X}$ is a fuzzy soft set-valued map $T_{\Upsilon}: X \longrightarrow I^{(E ; X)}$, defined by

$$
T_{\Upsilon}[x ; e](t)=\{t \in X:(\Upsilon x)(t) \geq \alpha\}, \alpha \in(0,1] .
$$

Notice that $I^{X} \longmapsto I^{(E ; X)}$ is embedding by $\Lambda \longrightarrow \Theta_{\Lambda}$, for every $\Lambda$ in $I^{X}$; where

$$
\Theta_{\Lambda}[x ; e](t)=\{t \in X: \Lambda(t) \geq e\} .
$$

Let $X$ be a metric space. For $x \in X$, consider two fuzzy soft sets $A, B \in I^{(E ; X)}$ and $e \in E$. Assume that $[A]_{E}^{e},[B]_{E}^{e} \in C B(X)$. Then define

$$
\begin{aligned}
& P_{E}^{e}(x, A)=\inf \left\{d(x, a): a \in[A]_{E}^{e}\right\} ; \\
& P_{E}^{e}(A, B)=\inf \left\{d(a, b): a \in[A]_{E}^{e}, b \in[B]_{E}^{e}\right\} ; \\
& P(A, B)=\sup _{e} P_{E}^{e}(A, B) ; \\
& D_{E}^{e}(A, B)=d_{E}^{H}\left([A]_{E}^{e},[B]_{E}^{e}\right) ;
\end{aligned}
$$

where

$$
d_{E}^{H}\left([A]_{E}^{e},[B]_{E}^{e}\right)=\max \left\{\sup _{a \in[A]_{E}^{e}} d\left(a,[B]_{E}^{e}\right), \sup _{b \in[B]_{E}^{e}} d\left(b,[A]_{E}^{e}\right)\right\} .
$$

Define a distance function $d_{E}^{\infty}: I^{(E ; X)} \times I^{(E ; X)} \longrightarrow \mathbb{R}$ by

$$
d_{E}^{\infty}\left([A]_{E}^{e},[B]_{E}^{e}\right)=\sup d_{E}^{H}\left([A]_{E}^{e},[B]_{E}^{e}\right) .
$$

Let $g: X \longrightarrow X$ be a single-valued mapping and $T: X \longrightarrow$ $I^{(E ; X)}$ be a fuzzy soft set-valued map. An element $x \in X$ is said to be:

(i) A fixed point of $g$ if $x=g x$. Denote by $\mathcal{F}_{i x}(g)$, the set of all fixed points of $g$.

(ii) Fuzzy soft fixed point of $T$ if $x \in[T x]_{E}^{e}$ for some $e \in E$. 
For $x, y \in X$, define

$$
\begin{gathered}
\bigsqcup(x, y)=\max \left\{d(x, y), d\left(x,[T x]_{E}^{e}\right), d\left(y,[T y]_{E}^{e}\right),\right. \\
\left.\frac{d\left(x,[T y]_{E}^{e}\right)+d\left(y,[T x]_{E}^{e}\right)}{2}, \frac{d\left(x,[T x]_{E}^{e}\right) d\left(y,[T y]_{E}^{e}\right)}{1+d(x, y)}\right\} \\
\coprod^{g}(x, y)=\max \{d(x, y), d(x, g x), d(y, g y), \\
\left.\frac{d(x, g y)+d(y, g x)}{2}, \frac{d(x, g x) d(y, g y)}{1+d(x, y)}\right\} . \\
\prod(x, y)=\min \left\{d\left(x,[T x]_{E}^{e}\right), d\left(y,[T x]_{E}^{e}\right),\right. \\
\frac{d\left(x,[T x]_{E}^{e}\right) d\left(y,[T y]_{E}^{e}\right)}{1+d(x, y)} . \\
\prod^{g}(x, y)=\min \left\{d(x, g x), d(y, g x), \frac{d(x, g x) d(y, g y)}{1+d(x, y)}\right\} .
\end{gathered}
$$

Throughout this paper, for $E=(0, \sigma), \sigma \geq 1$, the function $r: E \longrightarrow\left(\frac{1}{2}, 1\right)$ is defined by

$$
r(e)= \begin{cases}1, & \text { if } 0<e<\frac{1}{2} \\ 1-e, & \text { if } \frac{1}{2} \leq e<\frac{1}{\sigma} .\end{cases}
$$

Motivated by definitions 1.2 and 3 as well as Theorem 1.4, we give the next Definition.

Definition 2.6. A fuzzy soft set-valued map $T: X \longrightarrow I^{(E ; X)}$ is called Suzuki-type soft $(e, K)$-weak contraction iffor all $x, y \in X$ with $x \neq y$, there exist some $e \in E$ and $K \geq 0$ such that

$$
r(e) d\left(x,[T x]_{E}^{e}\right) \leq d(x, y)
$$

implies

$$
d_{E}^{H}\left([T x]_{E}^{e},[T y]_{E}^{e}\right) \leq e \coprod(x, y)+K \prod(x, y) .
$$

\subsection{Fuzzy Soft Fixed Points of Suzuki-type Soft $(e, K)$-Weak Contractions}

In this subsection, we first present fuzzy soft fixed theorem of Suzuki-type fuzzy soft set-valued $(e, K)$-weak contractions and then obtain some associated consequences.

Theorem 2.7. Let $(X, d)$ be a complete metric space and $T$ : $X \longrightarrow I^{(E ; X)}$ a Suzuki-type fuzzy soft set-valued $(e, K)$-weak contraction. If for some $x \in X$, there exists $e \in E$ such that $[T x]_{E}^{e}$ is a nonempty closed and bounded subset of $X$, then $\mathcal{F}_{\text {ix }}(T) \neq \emptyset$.

Proof. Let $e_{1} \in E$ be such that $0<e \leq e_{1}<\frac{1}{\sigma}, x_{1} \in X$ and $\rho=\frac{1}{\sqrt{e}}$. Then by hypothesis, $\left[\text { T }_{1}\right]_{E}^{e}$ is nonempty. Therefore, we can find $x_{2} \in X$ such that $x_{2} \in\left[T x_{1}\right]_{E}^{e}$. Since $\rho>1$, choose $x_{3} \in\left[T x_{2}\right]_{E}^{e}$ such that

$$
d\left(x_{2}, x_{3}\right) \leq \rho d_{E}^{H}\left(\left[T x_{1}\right]_{E}^{e},\left[T x_{2}\right]_{E}^{e}\right) .
$$

If $x_{1}=x_{2}$, then $x_{1} \in\left[T x_{1}\right]_{E}^{e}$ for some $e \in E$, and the theorem is proved. Assume that $x_{1} \neq x_{2}$. Since $r(e) \leq 1$, therefore,

$$
r(e) d\left(x_{1},\left[T x_{1}\right]_{E}^{e}\right) \leq d\left(x_{1},\left[T x_{1}\right]_{E}^{e}\right) \leq d\left(x_{1}, x_{2}\right)
$$

implies

If $d\left(x_{1}, x_{2}\right) \leq d\left(x_{2}, x_{3}\right)$, then from (5), we have

$$
d\left(x_{2}, x_{3}\right) \leq \sqrt{e} d\left(x_{2}, x_{3}\right)<d\left(x_{2}, x_{3}\right),
$$

a contradiction. Hence, $d\left(x_{1}, x_{2}\right)>d\left(x_{2}, x_{3}\right)$ and (5) becomes

$$
d\left(x_{2}, x_{3}\right) \leq \sqrt{e} d\left(x_{1}, x_{2}\right) \leq \sqrt{e_{1}} d\left(x_{1}, x_{2}\right) .
$$

Continuing in this fashion, we generate a sequence $\left\{x_{n}\right\}_{n \in \mathbb{N}}$ in $X$ such that $x_{n+1} \in\left[T x_{n}\right]_{E}^{e}$ and

$$
d\left(x_{n}, x_{n+1}\right) \leq \sqrt{e_{1}} d\left(x_{n-1}, x_{n}\right),
$$

from which we have

$$
\sum_{n=1}^{\infty} d\left(x_{n}, x_{n+1}\right) \leq \sum_{n=1}^{\infty}\left(\sqrt{e_{1}}\right)^{n-1} d\left(x_{1}, x_{2}\right)<\infty .
$$

By a standard argument, we conclude that $\left\{x_{n}\right\}_{n \in \mathbb{N}}$ is a Cauchy sequence in $\mathrm{X}$. The completeness of $X$ implies that there exists $u \in X$ such that $x_{n} \longrightarrow u$ as $n \longrightarrow \infty$.

Claim: for all $u \neq z$,we have

$$
d\left(u,[T z]_{E}^{e}\right) \leq e \max \left\{d(u, z), d\left(z,[T z]_{E}^{e}\right)\right\} .
$$

Since $x_{n} \longrightarrow u$ as $n \longrightarrow \infty$, there exists a positive integer $m$ such that

$$
d\left(x_{n}, u\right) \leq \frac{1}{3} d(u, z), \text { for all } n \geq m .
$$

Given that $x_{n+1} \in\left[T x_{n}\right]_{E}^{e}$, we get

$$
\begin{aligned}
r(e) d\left(x_{n},\left[T x_{n}\right]_{E}^{e}\right) & \leq d\left(x_{n},\left[T x_{n}\right]_{E}^{e}\right) \\
& \leq d\left(x_{n}, x_{n+1}\right) \\
& \leq d\left(x_{n}, u\right)+d\left(u, x_{n+1}\right) \\
& \leq \frac{2}{3} d(u, z) .
\end{aligned}
$$

Thus, for $n \geq m$, we have

Hence,

$$
r(e) d\left(x_{n},\left[T x_{n}\right]_{E}^{e}\right) \leq d\left(x_{n}, z\right)
$$

implies

From (9), we have

As $n \longrightarrow \infty$ in (10), we obtain

$$
\begin{aligned}
d\left(u,[T z]_{E}^{e}\right) \leq & e \max \left\{d(u, z), d\left(z,[T z]_{E}^{e}\right), \frac{d\left(u,[T z]_{E}^{e}\right)+d(u, z)}{2}\right\} \\
& +K \min \{0, d(u, z)\} . \\
\leq & e \max \left\{d(u, z), d\left(z,[T z]_{E}^{e}\right)\right\} .
\end{aligned}
$$


Now, to show that $u \in[T u]_{E}^{e}$ for some $e \in E$, we consider the following two possible cases:

Case(i): $0<e<\frac{1}{2}$.

Suppose that for all $e \in E, u \neq p, u \notin[T u]_{E}^{e}$ and $p \in[T u]_{E}^{e}$ such that $d(p, u)<d\left(u,[T u]_{E}^{e}\right)$. Setting $z=p$ in (6), we have

$$
d\left(u,[T p]_{E}^{e}\right) \leq e \max \left\{d(u, p), d\left(p,[T p]_{E}^{e}\right) .\right\}
$$

Now,

$$
r(e) d\left(u,[T u]_{E}^{e}\right) \leq d\left(u,[T u]_{E}^{e}\right) \leq d(u, p)
$$

implies

Suppose that $d(u, p) \leq d\left(p,[T p]_{E}^{e}\right)$, then from (12), we have

$$
d\left(p,[T p]_{E}^{e}\right) \leq e d\left(p,[T p]_{E}^{e}\right)<d\left(p,[T p]_{E}^{e}\right),
$$

a contradiction. Hence, $d(u, p)>d\left(p,[T p]_{E}^{e}\right)$, and

$$
d\left(p,[T p]_{E}^{e}\right) \leq e d(u, p)<d(u, p) .
$$

Therefore, (11) becomes $d\left(u,[T p]_{E}^{e}\right) \leq e d(u, p)$. Consequently,

$$
\begin{aligned}
d\left(u,[T u]_{E}^{e}\right) & \leq d\left(u,[T p]_{E}^{e}\right)+d_{E}^{H}\left([T p]_{E}^{e},[T u]_{E}^{e}\right) \\
& \leq d\left(u,[T p]_{E}^{e}\right)+e \max \left\{d(u, p), d\left(p,[T p]_{E}^{e}\right)\right\} \\
& \leq e d(u, p)+e d(u, p)=2 e d(u, p) \\
& <d(u, p)<d\left(u,[T u]_{E}^{e}\right)
\end{aligned}
$$$$
\text { (in view of assumption) }
$$

yields a contradiction. Therefore, $u \in[T u]_{E}^{e}$ for some $e \in E$.

Case(ii): $\frac{1}{2} \leq e<\frac{1}{\sigma}$.

For this, we shall prove that

holds for all $z \in X$ with $z \neq u$. Now, for all $m \in \mathbb{N}$, there exists $v_{m} \in[T z]_{E}^{e}$ such that

$$
d\left(u, v_{m}\right) \leq d\left(u,[T z]_{E}^{e}\right)+\frac{1}{5 m} d(u, z) .
$$

Thus, we have

$$
\begin{aligned}
d\left(z,[T z]_{E}^{e}\right) & \leq d\left(z, v_{m}\right) \\
& \leq d(z, u)+d\left(u, v_{m}\right) \\
& \leq d(z, u)+d\left(u,[T z]_{E}^{e}\right)+\frac{1}{5 m} d(z, u) .
\end{aligned}
$$

Using (6), we have

If $d(z, u)>d\left(z,[T z]_{E}^{e}\right)$, then from (15), we get

$$
\begin{aligned}
d\left(z,[T z]_{E}^{e}\right) & \leq d(u, z)+e d(u, z)+\frac{1}{5 m} d(u, z) \\
& =\left[(1+e)+\frac{1}{5 m}\right] d(u, z),
\end{aligned}
$$

from which we have

$$
\left[\frac{1}{1+e}\right] d\left(z,[T z]_{E}^{e}\right) \leq\left[1+\frac{1}{5(1+e) m}\right] d(u, z) .
$$

Using $r(e)=1-e$, we get

As $m \longrightarrow \infty$ in (16), we have

$$
r(e) d\left(z,[T z]_{E}^{e}\right) \leq d(u, z) .
$$

On the other hand, if $d(u, z)<d\left(z,[T z]_{E}^{e}\right)$, then (15) gives

$$
d\left(z,[T z]_{E}^{e}\right) \leq d(u, z)+e d\left(z,[T z]_{E}^{e}\right)+\frac{1}{5 m} d(u, z),
$$

which yields

$$
(1-e) d\left(z,[T z]_{E}^{e}\right) \leq\left(1+\frac{1}{5 m}\right) d(u, z) .
$$

As $m \longrightarrow \infty$ in (17), we have

$$
(1-e) d\left(z,[T z]_{E}^{e}\right) \leq d(u, z) .
$$

This shows that $r(e) d\left(z,[T z]_{E}^{e}\right) \leq d(u, z)$, which, by Definition 2.6, implies (14). Moreover, since $x_{n+1} \neq x_{n}$ for all $n \in \mathbb{N}$, then $u \neq x_{n+1}$. Therefore, setting $x_{n}=z$ in (14), we have

As $n \longrightarrow \infty$ in (18), we have

Since $1-e>0$, therefore, (19) implies that $d\left(u,[T u]_{E}^{e}\right)=0$, and consequently, $u \in[T u]_{E}^{e}$, for some $e \in E$.

Example 2.8. Let $X=[-40,60], E=(0,30], e \in E$ be arbitrary and $d: X \times X \longrightarrow[0, \infty)$ be defined by

$$
d(x, y)=|x-y|, \text { for all } x, y \in X .
$$

Define the mapping $T: X \longrightarrow I^{(E ; X)}$ by

$$
T[x ; e](t)= \begin{cases}\Delta_{1}, & \text { if } 0 \leq t \leq \frac{x}{30} \\ \Delta_{2}, & \text { if } \frac{x}{30}<t \leq \frac{x}{10} \\ \Delta_{3}, & \text { if } \frac{x}{10}<t \leq 60\end{cases}
$$

where

$$
\Delta_{1}= \begin{cases}0.2, & \text { if } 0<e_{1} \leq 10 \\ 0.5, & \text { if } 10<e_{2} \leq 20 \\ 0.8, & \text { if } 20<e_{3} \leq 30 .\end{cases}
$$

$$
\Delta_{2}= \begin{cases}0.7, & \text { if } 0<e_{1} \leq 10 \\ 0.9, & \text { if } 10<e_{2} \leq 20 \\ 0, & \text { if } 20<e_{3} \leq 30\end{cases}
$$

$$
\Delta_{3}= \begin{cases}0.4, & \text { if } 0<e_{1} \leq 10 \\ 0.6, & \text { if } 10<e_{2} \leq 20 \\ 0.2, & \text { if } 20<e_{3} \leq 30 .\end{cases}
$$


If $e=0.8$, then

$$
\begin{aligned}
{\left[T\left[x ; e_{1}\right]\right]_{E}^{0.8}=} & \left\{t \in X: T\left[x ; e_{1}\right](t) \geq 0.8\right\}=\emptyset . \\
{\left[T\left[x ; e_{2}\right]\right]_{E}^{0.8} } & =\left\{t \in X: T\left[x ; e_{2}\right](t) \geq 0.8\right\} \\
& =\left(\frac{x}{30}, \frac{x}{10}\right] . \\
{\left[T\left[x ; e_{3}\right]\right]_{E}^{0.8} } & =\left\{t \in X: T\left[x ; e_{3}\right](t) \geq 0.8\right\} \\
& =\left[0, \frac{x}{30}\right] .
\end{aligned}
$$

Therefore,

$$
\begin{aligned}
{[T x]_{E}^{e} } & =\bigcup_{e \in E}[T[x ; e]]_{E}^{e} \\
& =\left[0, \frac{x}{10}\right] .
\end{aligned}
$$

Note that $T$ is a Suzuki-type fuzzy soft set-valued $(e, K)$-weak contraction with $e=0.8$ and $K=0$. In particular, for $x=40$ and $y=50$,

$$
\begin{aligned}
r(e) d\left(x,[T x]_{E}^{e}\right) & =r(0.8) d\left(40,[T 40]_{E}^{0.8}\right) \\
& =(0.2) d(40,[0,4])=7.2 \\
& \leq d(40,50)=10
\end{aligned}
$$

implies

$$
\begin{array}{r}
d_{E}^{H}\left([T 40]_{E}^{0.8},[T 50]_{E}^{0.8}\right)=d_{E}^{H}([0,4],[0,5])=1 \\
\leq 118=(0.8) \max \left\{d(40,50), d\left(40,[T 40]_{E}^{0.8}\right), d\left(50,[T 50]_{E}^{0.8}\right),\right.
\end{array}
$$$$
\left.\frac{d\left(40,[T 40]_{E}^{0.8}\right)+d\left(50,[T 40]_{E}^{0.8}\right)}{2}, \frac{d\left(40,[T 40]_{E}^{0.8}\right) d\left(50,[T 50]_{E}^{0.8}\right)}{1+d(40,50)}\right\}
$$

Consequently, Theorem 2.7 can be applied to find $0 \in X$ such that $0 \in[T 0]_{E}^{0.8}$.

Corollary 2.9. Let $(X, d)$ be a complete metric space and $T$ : $X \longrightarrow I^{(E ; X)}$ a fuzzy soft set-valued map. Assume that for $x, y \in$ $X$ with $x \neq y$, there exists $e \in E$ such that $[T x]_{E}^{e}$ is a nonempty closed and bounded subsets of $X$. If

$$
r(e) d\left(x,[T x]_{E}^{e}\right) \leq d(x, y)
$$

implies

$$
d_{E}^{\infty}\left([T x]_{E}^{e},[T y]_{E}^{e}\right) \leq e \coprod(x, y)+K \prod(x, y),
$$

then $\mathcal{F}_{i x}(T) \neq \emptyset$.

Proof. Since $d_{E}^{H}\left([T x]_{E}^{e},[T y]_{E}^{e}\right) \leq d_{E}^{\infty}\left([T x]_{E}^{e},[T y]_{E}^{e}\right)$, then by Theorem 2.7, the conclusion holds.

Corollary 2.10. Let $(X, d)$ be a complete metric space and $T$ : $X \longrightarrow I^{(E ; X)}$ a fuzzy soft set-valued map. Assume that for $x, y \in$ $X$ with $x \neq y$, there exists $e \in E$ such that $[T x]_{E}^{e}$ is a nonempty closed and bounded subsets of $X$. If

$$
r(e) d\left(x,[T x]_{E}^{e}\right) \leq d(x, y)
$$

implies

$$
d_{E}^{H}\left([T x]_{E}^{e},[T y]_{E}^{e}\right) \leq e \coprod(x, y),
$$

Then $\mathcal{F}_{i x}(T) \neq \emptyset$.
Corollary 2.11. Let $(X, d)$ be a complete metric space and $T$ : $X \longrightarrow I^{(E ; X)}$ a soft set-valued map. If there exist positive constants $a, b, c$ with $e=a+b+c<1, K \geq 0$ such that for $x, y \in X$ with $x \neq y,[T x]_{E}^{e}$ is a nonempty closed and bounded subsets of $X$ and

$$
r(e) d\left(x,[T x]_{E}^{e}\right) \leq d(x, y)
$$

implies

$d_{E}^{H}\left([T x]_{E}^{e},[T y]_{E}^{e}\right) \leq a d(x, y)+b d\left(x,[T x]_{E}^{e}\right)+c d\left(d\left(y,[T y]_{E}^{e}\right)\right)+K \prod(x, y)$.

Then $\mathcal{F}_{i x}(T) \neq \emptyset$.

Corollary 2.12. Let $(X, d)$ be a complete metric space and $g$ : $X \longrightarrow X$ a single-valued mapping. Assume that there exists $0<e<1$ and $K \geq 0$ such that for $x, y \in X$ with $x \neq y, g(X)$ is a nonempty closed and bounded subset of $X$. If

$$
r(e) d(x, g x) \leq d(x, y)
$$

implies

$$
d(g x, g y) \leq e \coprod^{g}(x, y)+K \prod^{g}(x, y),
$$

then $g$ has a unique fixed point.

Proof. The existence of the fixed point of $g$ follows from Theorem 2.7. For uniqueness, suppose that there exist $u_{1}, u_{1} \in X$ with $u_{1} \neq u_{2}$ such that $g u_{1}=u_{1}$ and $g u_{2}=u_{2}$. Then

$$
r(e) d\left(u_{1}, g u_{1}\right) \leq d\left(u_{1}, g u_{1}\right)=d\left(u_{1}, u_{1}\right)=0 \leq d\left(u_{1}, u_{2}\right)
$$

implies

$$
\begin{aligned}
d\left(u_{1}, u_{2}\right)= & d\left(g u_{1}, g u_{2}\right) \\
\leq & e \max \left\{d\left(u_{1}, u_{2}\right), d\left(u_{1}, g u_{1}\right), d\left(u_{2}, g u_{2}\right)\right. \\
& \left.\frac{d\left(u_{1}, g u_{2}\right)+d\left(u_{2}, g u_{1}\right)}{2}, \frac{d\left(u_{1}, g u_{1}\right) d\left(d\left(u_{2}, g u_{2}\right)\right)}{1+d\left(u_{1}, u_{2}\right)}\right\} \\
& +K \min \left\{d\left(u_{1}, g u_{1}\right), d\left(u_{2}, g u_{1}\right), \frac{d\left(u_{1}, g u_{1}\right) d\left(u_{2}, g u_{2}\right)}{1+d\left(u_{1}, u_{2}\right)}\right. \\
\leq & e \max \left\{d\left(u_{1}, u_{2}\right), d\left(u_{1}, u_{1}\right), d\left(u_{2}, u_{2}\right)\right. \\
& \left.\frac{d\left(u_{1}, u_{2}\right)+d\left(u_{1}, u_{2}\right)}{2}, \frac{d\left(u_{1}, u_{1}\right) d\left(u_{2}, u_{2}\right)}{1+d\left(u_{1}, u_{2}\right)}\right\} \\
& +K \min \left\{d\left(u_{1}, u_{1}\right), d\left(u_{1}, u_{2}\right), \frac{d\left(u_{1}, u_{1}\right) d\left(u_{2}, u_{2}\right)}{1+d\left(u_{1}, u_{2}\right)}\right\} \\
\leq & e \max \left\{d\left(u_{1}, u_{2}\right), 0\right\}+K(0) \\
\leq & e d\left(u_{1}, u_{2}\right)
\end{aligned}
$$

a contradiction. Therefore $u_{1}=u_{2}$.

Next, we present a local fuzzy soft fixed point theorem for Suzuki-type soft $(e, K)$-weak contractions. First, recall that an open ball with radius $r>0$, centered at $x_{0}$ in a metric space $X$, is given by

$$
B_{r}\left(x_{0}\right)=\left\{x \in X: d\left(x, x_{0}\right)<r\right\} .
$$


Theorem 2.13. Let $(X, d)$ be a complete metric space,

$T: B_{r}\left(x_{0}\right) \longrightarrow I^{(E ; X)}$ be a Suzuki-type fuzzy soft set-valued $(e, K)$-weak contraction. Assume that for some $x \in X$, there exists $e \in E$ such that $[T x]_{E}^{e}$ is a nonempty closed and bounded subset of $X$ and

$$
d\left(x_{0},\left[T x_{0}\right]_{E}^{e}\right)<(1-e) r .
$$

Then $\mathcal{F}_{i x}(T) \neq \emptyset$.

Proof. Let $0<\eta<r$ be such that $0<(1-e)(1+\sqrt{e}) \leq$ $\frac{1}{1+\eta}, B_{\eta}^{*}\left(x_{0}\right) \subset B_{r}\left(x_{0}\right)$ and $d\left(x_{0},\left[T x_{0}\right]_{E}^{e}\right)<(1-e) \eta$. Therefore, $(1-e) \eta-d\left(x_{0},\left[T x_{0}\right]_{E}^{e}\right)>0$. Choose $\gamma=(1-e) \eta-$ $d\left(x_{0},\left[T x_{0}\right]_{E}^{e}\right)>0$; then there exists $x_{1} \in\left[T x_{0}\right]_{E}^{e}$ such that $d\left(x_{0}, x_{1}\right)<d\left(x_{0},\left[T x_{0}\right]_{E}^{e}\right)+\gamma$. Thus, $d\left(x_{0}, x_{1}\right)<(1-e) \eta$. Now, for $\rho=\frac{1}{\sqrt{e}}$ and $x_{1} \in\left[T x_{0}\right]_{E}^{e}$, there exists $x_{2} \in\left[T x_{1}\right]_{E}^{e}$ such that $d\left(x_{1}, x_{2}\right) \leq \rho d_{E}^{H}\left(\left[T x_{0}\right]_{E}^{e},\left[T x_{1}\right]_{E}^{e}\right)$. Noting that

$$
r(e) d\left(x_{0},\left[T x_{0}\right]_{E}^{e}\right) \leq r(e) d\left(x_{0}, x_{1}\right) \leq d\left(x_{0}, x_{1}\right), \text { we have }
$$

If $d\left(x_{0}, x_{1}\right) \leq d\left(x_{1}, x_{2}\right)$, then (20) gives

$$
d\left(x_{1}, x_{2}\right) \leq \sqrt{e} d\left(x_{1}, x_{2}\right)<d\left(x_{1}, x_{2}\right),
$$

a contradiction. Hence, $d\left(x_{0}, x_{1}\right)>d\left(x_{1}, x_{2}\right)$ and

$$
d\left(x_{1}, x_{2}\right)<\sqrt{e} d\left(x_{0}, x_{1}\right)<\sqrt{e}(1-e) \eta .
$$

Notice that by assumption and the triangle inequality, we have

$$
\begin{aligned}
d\left(x_{0}, x_{2}\right) & \leq d\left(x_{0}, x_{1}\right)+d\left(x_{1}, x_{2}\right) \\
& <(1-e) \eta+\sqrt{e}(1-e) \eta \\
& \leq \frac{\eta}{1+\eta}<\eta .
\end{aligned}
$$

Hence, $x_{2} \in B_{\eta}\left(x_{0}\right)$. Continuing this process recursively, we generate a sequence $\left\{x_{n}\right\}_{n \in \mathbb{N}}$ such that

(a) $x_{n} \in B_{\eta}\left(x_{0}\right)$ for all $n \in \mathbb{N}$;

(b) $x_{n} \in\left[T x_{n}\right]_{E}^{e}$ for each $n \in \mathbb{N}$;

(c) $d\left(x_{n}, x_{n+1}\right) \leq(\sqrt{e})^{n}(1-e) \eta$ for all $n \in \mathbb{N}$.

From (c), it follows by usual arguments that $\left\{x_{n}\right\}_{n \in \mathbb{N}}$ is a Cauchy sequence and converges to some $u \in B_{r}\left(x_{0}\right)$. From here, following the steps in the proof of theorem 2.7, we conclude that $\mathcal{F}_{i x}(T) \neq \emptyset$.

\subsection{Consequences}

Here, we show that there is a link between multi-valued mappings and fuzzy soft set-valued maps by obtaining some existing results as consequences of our result.

Corollary 2.14. (See [16, Theorem 2.1]) Let $(X, d)$ be a complete metric space and $T: X \longrightarrow C B(X)$ be a multi-valued mapping. Assume that there exists an $\alpha \in[0,1)$ such that for all $x, y \in X$,

$$
r(\alpha) d(x, T x) \leq d(x, y)
$$

implies

$$
H(T x, T y) \leq \alpha d(x, y)
$$

where the mapping $r:[0,1) \longrightarrow(0,1]$ is defined by

$$
r(\alpha)= \begin{cases}1, & \text { if } 0 \leq \alpha \leq 1 \\ 1-\alpha, & \text { if } \frac{1}{2} \leq \alpha<1 .\end{cases}
$$

Then there exists $u \in X$ such that $u \in T u$.

Proof. Let $E:=(0,1)$ be a parameter set and $e \in E$ be arbitrary. Consider a mapping $\varpi: X \longrightarrow E$ and a fuzzy soft set-valued map $\digamma: X \longrightarrow I^{(E ; X)}$ defined by

$$
\digamma[x ; e](t)= \begin{cases}\varpi(x), & \text { if } t \in T x \\ 0, & \text { if } t \notin T x .\end{cases}
$$

Then

$$
\begin{aligned}
{[\digamma x]_{E}^{e} } & =\bigcup_{e \in E}\{t \in X: \digamma[x ; e](t) \geq \varpi(x)=e\} \\
& =T x .
\end{aligned}
$$

Therefore,

$$
\begin{aligned}
d(x, y) & \geq r(\alpha) d(x, T x) \\
& =r(\alpha) d\left(x,[\digamma x]_{E}^{e}\right) .
\end{aligned}
$$

Thus, Theorem 2.7 can be applied to find $u \in X$ such that $u \in$ Tu.

From Theorem 2.7 and using the method of proof of Corollary 2.14, we can also derive the following results.

Corollary 2.15. (See [19]) Let $(X, d)$ be a complete metric space and $T: X \longrightarrow C B(X)$ be a multi-valued mapping. Assume that there exists $\alpha \in[0,1)$ such that $r(\alpha) d(x, T x) \leq d(x, y)$ implies

$$
H(T x, T y) \leq \alpha \max \{d(x, y), d(x, T x), d(y, T y)\}
$$

for all $x, y \in X$, where the function $r$ is defined as in Corollary 2.14. Then, there exists $u \in X$ such that $u \in T u$.

Corollary 2.16. (See [9]) Let $(X, d)$ be a complete metric space and $T: X \longrightarrow C B(X)$ be a multi-valued mapping. Let the function $r:[0,1) \longrightarrow[0,1)$ be as defined in Corollary 2.14. Assume that there exists $a(x, y) \in[0,1)$ such that

$$
\begin{aligned}
& r(\alpha) d(x, T x) \leq d(x, y) \text { implies } \\
& H(T x, T y) \leq a(x, y)[d(x, T y)+d(y, T x)]
\end{aligned}
$$

for all $x, y \in X$. Then there exists $u \in X$ such that $u \in T u$.

Corollary 2.17. Let $(X, d)$ be a complete metric space and $T$ : $X \longrightarrow C B(X)$ a multi-valued mapping. Assume that there exist constants $\alpha \in[0,1)$ and $K \geq 0$ such that for all $x, y$ in $X$ with $x \neq y$, if

$$
r(\alpha) d(x, T x) \leq d(x, y)
$$

implies

$$
H(T x, T y) \leq \alpha \coprod(x, y)+K \prod(x, y),
$$

where the function $r$ is as defined in Corollary 2.14, Then, there exists $u \in X$ such that $u \in T u$. 
Corollary 2.18. Let $(X, d)$ be a complete metric space and $T$ : $X \longrightarrow C B(X)$ a multi-valued mapping. Assume that there exist constants $\alpha \in[0,1)$ and $K \geq 0$ such that for all $x, y$ in $X$ with $x \neq y$, if

$$
r(\alpha) d(x, T x) \leq d(x, y)
$$

implies

$$
d_{\infty}(T x, T y) \leq \alpha \coprod(x, y)+K \prod(x, y),
$$

where the function $r$ is as defined in Corollary 2.14. Then, there exists $u \in X$ such that $u \in T u$.

In Corollary 2.17, if $T$ is taken as a single-valued mapping, then we have the next result.

Corollary 2.19. Let $(X, d)$ be a complete metric space and $T$ : $X \longrightarrow X$ be a single-valued mapping. Assume that there exist constants $\alpha \in[0,1)$ and $K \geq 0$ such that for all $x, y$ in $X$ with $x \neq y$, if

$$
r(\alpha) d(x, T x) \leq d(x, y)
$$

implies

$$
d(T x, T y) \leq \alpha \coprod(x, y)+K \prod(x, y),
$$

where the function $r$ is as defined in Corollary 2.14, Then, there exists a unique $u \in X$ such that $u=T u$.

\subsection{Application in Homotopy}

In this section, we apply Theorem 2.13 to prove a homotopy result. First, for convenience, we recall the following familiar definitions.

Definition 2.20. A relation $\leq$ is a total order on a set $U$ if for all $s, t, u \in U$, the following conditions hold:

(i) Reflexivity: $s \leq s$;

(ii) Antisymmetry: if $s \leq t$ and $t \leq s$, then $s=t$;

(iii) Transitivity: if $s \leq t$ and $t \leq u$, then $s \leq u$;

(iv) Comparability: for every $s, t \in U$, either $s \leq t$ or $t \leq s$.

Recall that if the set $U$ satisfies only the axioms (i) - (iii), then it is said to be partially ordered. In what follows, we shall call a totally ordered set a chain.

Lemma 2.21. (Kuratowski-Zorn's Lemma) If U is any nonempty partially ordered set in which every chain has an upper bound, then $U$ has a maximal element.

Definition 2.22. Let $X_{1}$ and $X_{2}$ be any two topological spaces and $\pi, \omega: X_{1} \longrightarrow X_{2}$ a continuous functions. A function $H$ : $X_{1} \times[0,1] \longrightarrow X_{2}$ such that if $x \in X_{1}$, then $H(u, 0)=\pi(u)$ and $H(u, 1)=\omega(u)$, is called a homotopy between $\pi$ and $\omega$.

We shall denote the boundary of a set $U$ by $B d(U)$.

Theorem 2.23. Let $(X, d)$ be a complete metric space and $U$ be an open subset of $X$. If $M: \bar{U} \times[0,1] \longrightarrow I^{(E ; X)}$ satisfies the following conditions: (hom-2) $M(., t): \bar{U} \longrightarrow I^{(E ; X)}$ is a Suzuki-type soft set-valued $(e, K)$-weak contraction for all $t \in[0,1]$;

(hom-3) there exist a nondecreasing function $f:[0,1] \longrightarrow \mathbb{R}$ such that

$$
\begin{aligned}
& d_{E}^{H}(M(u, t), M(u, s)) \leq|f(t)-f(s)| \text { for all } s, t \in[0,1] \\
& \text { and each } u \in \bar{U}
\end{aligned}
$$

(hom-4) $M: \bar{U} \times[0,1] \longrightarrow I^{(E ; X)}$ is closed and bounded.

Then $M(., 0)$ has a fixed point.

Proof. Assume $p$ is a fixed point of $M(., 0)$. Then by (hom-1), $p \in U$. Consider the set $\bigwedge$ given by

$$
\bigwedge=\{(t, u) \in[0,1] \times U: u \in M(u, t)\} .
$$

Notice that $(0, p) \in \bigwedge$; hence $\bigwedge \neq \emptyset$. Let $e \in E:=(0,1)$ and define a partial order $\leq$ on $\bigwedge$ as follows:

$$
(t, u) \leq(s, v) \text { if and only if } t \leq s \text { and } d(u, v) \leq \frac{2}{1-e}[f(s)-f(t)] .
$$

Suppose $\Omega$ is a chain of $\Lambda$ and $t^{*}:=\sup \{t:(t, u) \in \Omega\}$. Assume that $\left\{t_{n}, u_{n}\right\}$ is a sequence in $\Omega$ such that $\left(t_{n}, u_{n}\right) \leq\left(t_{n+1}, u_{n+1}\right)$ and $t_{n} \longrightarrow t^{*}$ as $n \longrightarrow \infty$. Then for all positive integers $m, n(m>$ $n)$,

$$
d\left(u_{m}, u_{n}\right) \leq \frac{2}{1-e}\left|f\left(t_{m}\right)-f\left(t_{n}\right)\right| .
$$

As $m, n \longrightarrow$ in (22), we get $d\left(u_{m}, u_{n}\right) \longrightarrow 0$. Hence, $\left\{u_{n}\right\}_{n \in \mathbb{N}}$ is a Cauchy sequence and converges to some $u^{*} \in X$. Since $M$ is closed and $u_{n} \in M\left(u_{n}, t_{n}\right)$, therefore, $u^{*} \in M\left(u^{*}, t^{*}\right)$. From condition (hom - 1), $u^{*} \in U$. Thus, $\left(t^{*}, u^{*}\right) \in \bigwedge$. Since $\Omega$ is a chain, therefore, $(t, u) \leq\left(t^{*}, u^{*}\right)$ for all $(t, u) \in \Omega$. In other words, $\left(t^{*}, u^{*}\right)$ is an upper bound of $\Omega$. Thus, by KuratowskiZorn Lemma's , $\bigwedge$ has a maximal element $\left(t_{0}, u_{0}\right)$. Next, we show that $t_{0}=1$. Suppose on the contrary that $t_{0}<1$. Let $r=\frac{2}{1-e}\left|f(t)-f\left(t_{0}\right)\right|>0$ with $t \in\left(t_{0}, 1\right]$ such that $B_{r}\left(u_{0}\right) \subset U$. Notice that by (hom-3),

$$
\begin{aligned}
d\left(u_{0}, M\left(u_{0}, t\right)\right) & \leq d\left(u_{0}, M\left(u_{0}, t_{0}\right)\right)+d_{E}^{H}\left(M\left(u_{0}, t_{0}\right), M\left(u_{0}, t\right)\right) \\
& \leq\left|f(t)-f\left(t_{0}\right)\right|=\frac{(1-e) r}{2}<(1-e) r .
\end{aligned}
$$

Therefore, $M(., t): B_{r}\left(u_{0}\right) \longrightarrow I^{(E ; X)}$ satisfies all the hypotheses of Theorem 2.13 for every $t \in[0,1]$. Consequently, there exists $u \in B_{r}\left(u_{0}\right)$ such that $u \in M(u, t)$, which implies that $(t, u) \in \wedge$ for all $t \in[0,1]$. Now,

$$
d\left(u_{0}, u\right) \leq r=\frac{2}{1-e}\left|f(t)-f\left(t_{0}\right)\right|,
$$

yields $\left(t_{0}, u_{0}\right)<(t, u)$, a contradiction to the fact that $\left(t_{0}, u_{0}\right)$ is maximal. Conversely, assume that $M(., 1)$ has a fixed point; then on similar steps as above, one can show that $M(., 0)$ has a fixed point. 


\section{Competing Interests}

The author declares that there is no competing interests.

\section{Acknowledgments}

The author is thankful to the editors and the anonymous reviewers for their valuable suggestions and comments on the manuscript.

\section{References}

[1] S. Banach," Sur les opérations dans les ensembles abstraits et leur application aux équations intégrales", Fund. math. 3 (1922) 133.

[2] A. Azam, M. Arshad \& I. Beg, "Banach contraction principle on cone rectangular metric spaces", Applicable Analysis and Discrete Mathematics 3 (2009) 236.

[3] A. Azam, B. Fisher \& M. Khan, "Common fixed point theorems in complex valued metric spaces" Numerical Functional Analysis and Optimization 32 (2011) 243.

[4] J. Caristi, "Fixed point theorems for mappings satisfying inwardness conditions", Transactions of the American Mathematical Society 215 (1976) 251.

[5] L. B. Ćirić, "A generalization of Banach's contraction principle", Proceedings of the American Mathematical society 45 (1974) 267.

[6] A. Meir \& E. Keeler, "A theorem on contraction mappings", Journal of Mathematical Analysis and Applications 28 (1969) 326.

[7] S. B. Nadler, "Multi-valued contraction mappings", Pacific Journal of Mathematics 30(1969) 475.

[8] A. Azam \& M. Arshad, "Kannan fixed point theorem on generalized metric spaces", The Journal of Nonlinear Sciences and Its Applications 1 (2008) 45.

[9] S.K. Chatterjea, "Fixed point theorems", Acad. Bulgare Sci. 25 (1972) 727.

[10] Y. Ibrahim, “ Common Fixed Point Theorems for Multivalued Generalized $F$-Suzuki-Contraction Mappings in Complete Strong $b$-Metric Spaces", Journal of the Nigerian Society of Physical Sciences 1 (2019) 88.

[11] I. A. Rus, "Generalized contractions and applications", Cluj University Press 4 (2001)8.

[12] T, Zamfirescu, "Fixed point theorems in metric spaces", Archiv der Mathematik 23 (1972) 292.
[13] V. Berinde, "Approximating fixed points of weak contractions using the Picard iteration", In Nonlinear Analysis 9(2004) 43.

[14] M. Berinde \& V. Berinde, "On a general class of multi-valued weakly Picard mappings", Journal of Mathematical Analysis and Applications 326 (2007) 772.

[15] N. Çağman \& S. Enginoğlu, "Soft set theory and uni-int decision making", European Journal of Operational Research 207 (2010) 848.

[16] D. Doric \& R. Lazovic, "Some Suzuki-type fixed point theorems for generalized multivalued mappings and applications", Fixed Point Theory and Applications 1 (2011) 40.

[17] Q. Feng \& Y. Zhou, "Soft discernibility matrix and its applications in decision making", Applied Soft Computing 24(2014) 749.

[18] R. Kannan, "Some results on fixed points-II', The American Mathematical Monthly, 76(1969) 405.

[19] M. Kikkawa, \& T. Suzuki, "Some similarity between contractions and Kannan mappings", Fixed Point Theory and Applications 2008 (2008) 649749.

[20] Z. Kong, L. Gao, \& L. Wang, "Comment on: A fuzzy soft set theoretic approach to decision making problems", Journal of computational and applied mathematics 223 (2009) 540.

[21] P. K. Maji, P. Biswas \& A. R. Roy. "Fuzzy soft sets", Journal of fuzzy mathematics 6(2001) 9.

[22] X. Yang, D. Yu, J. Yang \& C. Wu, "Generalization of soft set theory: from crisp to fuzzy case", In Fuzzy Information and Engineering 3(2007) 345.

[23] P. K. Maji, A. R. Roy, \& R. Biswas, "An application of soft sets in a decision making problem", Computers and Mathematics with Applications 44 (2002) 1077.

[24] Roy, A. R. \& Maji, P. K., "A fuzzy soft set theoretic approach to decision making problems", Journal of Computational and Applied Mathematics 203 (2007) 412.

[25] S. S. Mohammed \& A. Azam, "An algorithm for fuzzy soft set based decision making approach", Yugoslav Journal of Operations Research 30 (2020) 59.

[26] D. Molodtsov, "Soft set theory—first results", Computers and Mathematics with Applications 37 (1999) 19.

[27] Z. Pawlak, "Rough sets", International journal of computer and information sciences 11 (1982) 341.

[28] P. V. Subrahmanyam, "Remarks on some fixed point theorems related to Banach's contraction principle”, J. Math. Phys. Sci. 8 (1974) 445.

[29] T. Suzuki, "A generalized Banach contraction principle that characterizes metric completeness", Proceedings of the American Mathematical Society 136 (2008) 1861.

[30] L. A. Zadeh, "Fuzzy sets", Information and control 8 (1965) 338. 